\title{
Equation of State of Stellar Plasmas
}

\author{
Forrest J. Rogers \\ Lawrence Livermore National Laboratory, Livermore, CA 94550
}

\begin{abstract}
The equation of state (EOS) of astrophysical plasmas is, for a wide range of stars, nearly ideal; with only small non-ideal Coulomb corrections. Calculating the EOS of an ionizing plasma from a ground state ion, ideal gas model is easy, whereas, fundamental methods to include the small Coulomb corrections are difficult. Attempts to include excited bound states are also complicated by plasma screening and microfield phenomena that weaken and broaden these states. Nevertheless, the high quality of current observational data, particularly seismic, dictates that the best possible models should be used. The present article discusses these issues and describes how they are resolved by fundamental many-body quantum statistical methods. Particular emphasis is placed on the activity expansion method that is the basis of the OPAL opacity code. Some comparisons with standard methods are given.
\end{abstract}

\section{Abstract}

L'equation d'etat des plasmas astrophysiques est, pour un large domaine d'etoiles, pratiquement ideale; avec de petites corrections coulombiennes. Calculer l'equation d'etat d'un plasma ionise a partir d'un modele de gaz ideal d'ions dans leur etat fondamental est facile, alors que les methodes fondamentales pour inclure les petites corrections coulombiennes sont difficiles. Des tentatives pour inclure des etats lies excites sont aussi rendues difficiles par les effets d'ecran et le phenomene de microchamp qui affaiblissent et elargissent ces etats. Neanmoins, la haute qualite des observations actuelles, en particulier en sismologie, impose l'utilisation des tous meilleurs modeles. Dans cet article, nous discutons les possibilites et nous decrivons les methodes de physique statistique quantique du probleme a $\mathrm{N}$-corps pour resoudre ce probleme. La methode de developpe opacite OPAL. Nous presentons des comparaisons avec des methodes standards. 


\subsection{Introduction}

The density temperature range found in stars is very large. Nevertheless, it is frequently true that the Coulomb correlational effects are small. For example, the density temperature profile of a main sequence star is given approximately by constant values of $R=\rho / T_{\%}^{3}$, where $\rho$ is the density in $\mathrm{g} / \mathrm{cm}^{3}$ and $\mathrm{T}_{6}$ is the temperature in millions of degrees Kelvin. Main sequence stars are about $98 \%$ hydrogen and helium by mass with the remainder being mostly Carbon, Nitrogen, and Oxygen. Since $\log R$ is less than -1 for most stars, these two conditions limit Coulomb corrections to the equation of state to generally less than a few percent. For stars in more advanced states of evolution, e.g. white dwarf and neutron stars, $R$ can be much larger and the composition in extreme cases can be pure iron. The discussion in the current paper will be primarily aimed at understanding the EOS in stars where the Coulomb interactions are small to moderate. Saumon and Chabrier $(1992 ; 1991)$ have been concerned with dense hydrogen and helium equations of state.

The simple ionization equilibrium model introduced by Saha (1920) led to a revolution in stellar modeling and has proved to be adequate for many purposes. Saha's model assumes ideal gas conditions and uses only the ground state configuration in the ionization balance equations. For example, in the simple case of a non-degenerate hydrogen plasma it gives

$$
\frac{\rho_{e} \rho_{P}}{\rho_{H}}=\frac{g_{e} g_{p}}{g_{H}} \frac{\lambda_{H}^{3}}{\lambda_{e}^{3} \lambda_{p}^{3}} \exp \left(\beta E_{1 s}\right)
$$

where $\rho_{i}=N_{i} / V$ is the number density for ions of type $i=\{e, p, H\}$,

$$
\lambda_{i}=\left(2 \pi \hbar^{2} / m_{i} k T\right)^{1 / 2}
$$

is the thermal de Broglie wavelength, and $g_{e}, g_{p}$, and $g_{H}$ are the statistical weights. The corresponding pressure is

$$
\frac{P}{k T}=\sum_{i} \rho_{i}
$$

Even though, as already noted, the Coulomb corrections to equation (1) are generally small for most stars, it is necessary to have a precise theory to account for them. There are two reasons for this. Firstly, a more complete model has to include all excited states including possible plasma screening effects, as well as, the Coulomb coupling corrections. These 
effects are interrelated and, as a result, ad hoc approaches are prone to count the same term twice. Consequently, the corrections to the Saha equation obtained by ad hoc approaches can vary by substantial amounts (gauged by modeling sensitivity). Secondly, the modeling of currently available high precision observational data requires very accurate EOS data (Christensen-Dalsgaard and Däppen 1993). Modeling of helioseismological data, for example, requires derivatives of the EOS that are accurate to better than $1 \%$. This level of accuracy can only be expected from fundamental procedures.

Considerable effort has been devoted to the fundamental treatment of hydrogen plasmas. An in depth description of much of this work can be found in the books by Kraeft, Kremp, Ebeling, and Röpke 1986 and Ebeling, Kraeft, and Kremp 1976; see also DeWitt 1966, Krasnikov 1977. The more general problem of multi-component plasmas has received much less attention (Rogers 1991; Krasnikov and Kucherenko 1978; Ebeling 1974). A new approach for treating multi-component plasmas is described in the articles by Aluestuey and Perez (1992) and Aluestuey elsewhere this volume. Numerous papers have been devoted to phenomenological modeling of multi-component plasmas (McChesney 1964).

\subsection{Commonly Used Methods}

Typical stellar model calculations require the EOS for a large, variable set of temperature-density points and for variable composition. It is thus very desirable to have available an efficient model that allows online computation, or failing that, a model that can be used to readily produce tables. All such models in current use are based on free energy minimization methods. These approaches work in the chemical picture and deal explicitly with ions and atoms.

Eggleton, Faulkner, and Flannery (1973) developed an EOS that is computationally simple and suitable for online use (EFF). They introduced an ad hoc free energy term to produce pressure ionization at high density, i.e., when the interparticle separation is less than a bohr. This overcomes a well known shortcoming of the Saha equation, which predicts that hydrogen is $30 \%$ neutral in the solar center where temperatures are 100 times the binding energy of $\mathrm{H}$. Similar to Saha, EFF assume that ions and atoms are in their unperturbed ground states. The EFF method accounts for Fermi-Dirac statistics for electrons, but ignores Coulomb interactions. It can produce unphysical phase transitions when used outside its range of validity. 
In view of the need for high accuracy in the EOS, a number of attempts have been made to improve the EFF equation. ChristiansenDalsgaard and Däppen (1991) and Swensen, VandenBerg, Alexander, and Irwin (1994) have added a Debye-Hückel free energy term (see equation (22)), called the CEFF equation to indicate that the Debye Coulomb correction has been added. Swenson and Rogers (1993) also adjusted parameters in the CEFF equation in order to obtain improved agreement with the activity expansion method.

Going beyond the simple models to include the excited states and more correct Coulomb interaction terms is fraught with difficulties. For example, the internal partition function for an isolated atom is divergent, so that some method for cutting off the partition function must be introduced. Typically this is accomplished by assuming that the presence of other particles in the vicinity of a given atom (ion) confines the particle to a sphere of order of the ion sphere radius, a, or that it interacts with the plasma through a short-ranged screened potential, e.g., the Debye-Hückel potential. The resulting free energy is finite but discontinuous at plasma conditions such that a bound state is just entering the continuum. This behavior cannot be present in physically consistent models.

Mihalas, Hummer, and Däppen (Hummer and Mihalas 1988; Mihalas, Däppen and Hummer 1988; Däppen, Anderson, and Mihalas 1987) have presented an occupation probability formalism that is physically consistent and produces continuous free energies. It is commonly referred to as the MHD equation of state. Based on experimental measurements of level shifts (Goldsmith, Griem and Cohen 1984), they assume that the bound states of atoms and ions are unshifted by the plasma environment. They note that, if a configurational free energy, $f\left(V, T,\left\{n_{i}\right\}\right)$, that depends explicitly on the occupation numbers of the individual states is added to the ideal free energy terms, then the ratio of the occupation of a state $i$ of a given ion to the total occupation is given by

$$
n_{i} / n=\exp \left[-\beta\left(E_{i}+\partial f / \partial n_{i}\right)\right] / \tilde{Z}_{\text {int }}
$$

where

$$
\tilde{Z}_{\text {int }}=\Sigma_{i} \exp \left[-\beta\left(E_{i}+\partial f / \partial n_{i}\right)\right]
$$

plays the role of the partition function,

$$
\omega_{i}=\exp \left[-\beta \partial f / \partial n_{i}\right]
$$


is the occupation probability, $n_{i}$ is the occupation number for state $i$ and $n$ is the total occupation for a given species. The occupation probability is a measure of the number of bound states of type $i$ that are available to be occupied. The quantity $1-\omega_{i}$ is, thus, a measure of the fraction of total states that have been severely affected by plasma perturbations and no longer act like localized states. In order to make progress it is necessary to have either a good estimate for $f$ or a good estimate for $\omega_{i}$, whichever is easier. The MHD approach mixes the two possibilities. In the case of neutral particle interactions they use the free energy of a parameterized hard sphere gas to determine the occupation probability. For ion-ion interactions they use the electric microfield (Stark-ionization theory) to determine the occupation probability. For ion-neutral interactions they propose using a product of the two forms. The method is thus phenomenological, but uses experimental data to fit free parameters in the occupation probability function. The MHD method has been used in numerous stellar modeling sensitivity studies (Christensen-Dalsgaard and Däppen 1993; Dziembowski, Pamyatnykh, and Sienkiewicz 1992). A method closely related to the MHD approach has also been developed by Sevastyanenko (1985).

\subsection{Consistent Treatment of Bound and Scattering States}

The source of the discontinuity in free energy minimization methods is easily shown to be the result of an inconsistent treatment of bound and scattering states. The second virial coefficient is the leading term in the density expansion of the free energy. The quantum mechanical second virial coefficient can be expressed in the form (Uhlenbeck and Beth 1936; Beth and Uhlenbeck 1939)

$$
B_{i j}=B_{i j}^{b x}+B_{i j}^{c z}
$$

where

$$
B_{i j}^{b s}=-\sqrt{2} \lambda_{i j}^{3} z_{\text {int }}, \quad z_{\text {int }}=\sum_{n \ell}(2 \ell+1) e^{-\beta E_{N \ell}}
$$

and

$$
B_{i j}^{c s}=-\frac{\sqrt{2}}{\pi} \lambda_{i j}^{3} \sum_{\eta \ell}(2 \ell+1) \int_{0}^{\infty} d p \frac{d \delta_{\ell}(p)}{d p} e^{-\beta_{p}^{2} / 2 \mu_{i j}}
$$


In equations $(7,9) \mathrm{i}$ and $\mathrm{j}$ are the particle types, $\lambda_{i j}=\left(\hbar^{2} / 2 \mu_{i j} k T\right)^{1 / 2}, \mathrm{n}$ and $\ell$, are quantum numbers, the $E_{n} \ell$ are bound state energies, $\delta_{\ell}$ is the phase shift, $\mathrm{p}$ is the relative momentum, and $\mu_{\mathrm{ij}}$ is the reduced mass. The connection between bound and scattering states can readily be established by integrating equation (9) by parts and using the fact that the phase shift at zero energy is just $n \pi$ (Levinson 1949). Adding the zero energy term to equation 8 (Rogers, Graboske, and DeWitt 1971) gives

$$
B_{i j}^{b s}=-\sqrt{2} \lambda_{i j}^{3} \sum_{n \ell}(2 \ell+1)\left(e^{-\beta E_{n \ell}}-1\right)
$$

If now each exponential (Boltzmann) factor is expanded in powers of $\beta E_{n \ell}$ it is apparent that the first term in the expansion just removes the negative term in equation (10). In other words, an analytical term having the form of the most divergent term in $Z_{i n t}$ is not actually present in $B_{i j}$; at least at temperatures where the Boltzmann factors are near unity. The sum over the $\beta \mathrm{E}_{\mathrm{n} \ell}$ terms in the expansion of $\mathrm{B}_{1}^{\mathrm{bs}}$ is also divergent, but with a second integration by parts of equation (9) it too can be shown to be analytically missing from the total $B_{i j}$ at high temperature (Rogers 1979; 1977; Bolle 1989; 1987; Pisano and McKellar 1989). There are divergences in $B_{i j}$, but they are all in the final form for $B_{i j}^{c s}$ after the two integrations by parts. These two integrations by parts have effectively redefined the continuum such that it begins at $-k T$, rather than the usual zero of energy. The effective internal partition function is thus,

$$
Z_{\text {in }}^{e}=\sum_{n \ell}(2 \ell+1)\left(e^{-\beta E_{m}}-1+\beta E_{n \ell}\right)
$$

It is worthwhile noting that the value of $B_{i j}$ has not changed in this series of manipulations, rather it has been shown that the analytic properties of the complete set of states dictates a specific separation of $B_{i j}$ into effective bound and scattering state parts. This separation will play an important role in the many-body statistical mechanical methods to be described in the next section.

\subsection{Classical Density Expansion for Plasmas}

The natural way to treat reacting, multicomponent plasmas is in the grand canonical ensemble (Hill 1956). In this approach one views the system in terms of its fundamental constituents, so that bound complexes arise naturally from the theory. As a result, this approach is commonly referred to as the physical picture method. The standard procedure is to 
expand the pressure in terms of two body, three body clusters, etc., i.e., a cluster expansion. The same is true for plasmas, but the long range of the Coulomb potential introduces substantial complications. In addition, the quantum nature of electrons introduces degeneracy and exchange corrections. The attractive electron-ion interaction leads to short distance divergences in classical cluster coefficients, so that the use of quantum mechanical methods is essential. Graphical resummation procedures are required to remove the long-range divergences occurring in all cluster coefficients of plasmas. The divergences in $\mathrm{B}_{11}^{\mathrm{CS}}$, mentioned in the previous section, are only the simplest examples. A detailed description of the procedure is given elsewhere (Dashen, Ma, and Bernstein 1969; Kraeft, Kremp, Ebeling and Röpke 1986; Rogers 1981). For illustrative purposes it is much easier to consider the related procedure in the canonical ensemble, i.e., an expansion of the free energy in the density. For simplicity we also focus on classical procedures where possible.

In early graphical analysis of the classical one-component plasma, ie., heavy ions emmersed in a continuous neutralizing background of electrons, Mayer (1950) referred to the non-ideal free energy (aside from a factor VkT) as -S, so that,

$$
\left(F-F_{0}\right) / V k T=-S
$$

where

$$
S=\sum_{j=2}^{-} B_{j} \frac{\rho^{j}}{j-1}
$$

$B_{j}$ is the $j$ th virial coefficient. The classical second virial coefficient for the one component plasma (OCP) is given by

$$
B_{2}=-\frac{1}{2 V} \int d \vec{r}_{1} \cdot d \vec{r}_{2}\left(e^{-\beta u\left(r_{12}\right)}-1\right)=-2 \pi \int_{0}^{\infty}\left(e^{-\beta \mu(r)}-1\right) r^{2} d r
$$

where $r_{12} \equiv r=\left|\vec{r}_{2}-\vec{r}_{1}\right|$

The divergence in $\mathrm{B}_{2}$ is easily seen by expanding the classical Boltzmann factors in powers of $\beta u$. The leading term diverges as $r^{2}$, the second term as $r$, and the third term logarithmically as $r \rightarrow \infty$. The leading divergence posses no problem, since it is canceled by an opposing term provided by the neutralizing electron background; leaving the $(\beta u)^{2}$ as the leading 
divergence. In general the lowest order term from each of the virial coefficients is given by

$$
B_{j}=(-1)^{j}(j-1) ! \beta^{j} \int \ldots \int d \vec{r}_{1} \ldots d r_{j} u\left(r_{12}\right) u\left(r_{23}\right) \ldots u\left(r_{j 1}\right)
$$

The sum over the most divergent terms from each of the higher $\mathbf{B}_{\mathbf{j}}$; i.e., those that have just one power of the potential turned on between each pair of particles, can be represented graphically as shown in Figure 1.

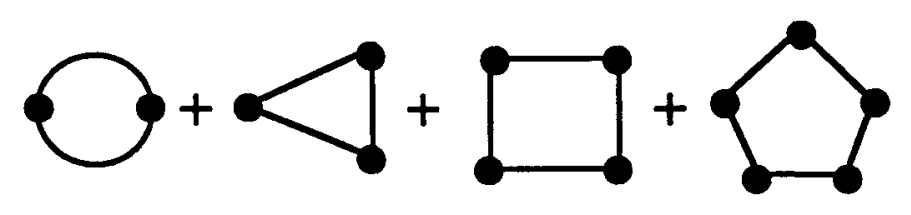

Fig. 1 Graphic illustration of the classical ring sum for the OCP. Solid circles represent ions. A diagram involving $\mathbf{n}$ ions comes from $B_{n}$.

Due to their topology, they are known as ring diagrams. The result of the summation over ring diagrams is

$$
S_{\text {ring }}=S_{D H}=\sum_{j} \rho^{j} B_{j}=\rho \Lambda / 3
$$

where

$$
\Lambda=Z^{2} e^{2} / k T \lambda_{D}
$$

and

$$
\lambda_{D}=\left(k T / 4 \pi Z^{2} e^{2} \rho\right)^{1 / 2}
$$

The pressure for a $v$ component plasma, corresponding to multicomponent generalization of equation (12), is

$$
P / k T=\sum_{v} \rho_{v}+S-\sum_{v} \rho_{v} \frac{\partial S}{\partial \rho_{v}}
$$


Using equation (16) this gives in the Debye-Hückel approximation for the OCP

$$
P / k T=\rho\left(1-\frac{\Lambda}{6}\right)
$$

The Debye-Hückel correction is just the leading term in an infinite expansion analogous to the virial expansion for an ordinary gas. However due to the Coulomb modifications it involves $\rho^{3 / 2}$ rather than $\rho^{2}$ (see equation 16). The next higher term in the expansion is obtained by summing over the next most divergent diagrams, the three rung ladder diagrams; so named by their ladder-like quantum mechanical form. Figure 2 shows (in the classical limit) how one rung of the three rung ladder diagram is screened by summing over chains, of ever increasing length, that have one power of the potential, $\beta u$, turned on between each pair of ions. Similar summations produce screening in the remaining two rungs of the diagram (often called watermelon diagrams in classical mechanics).

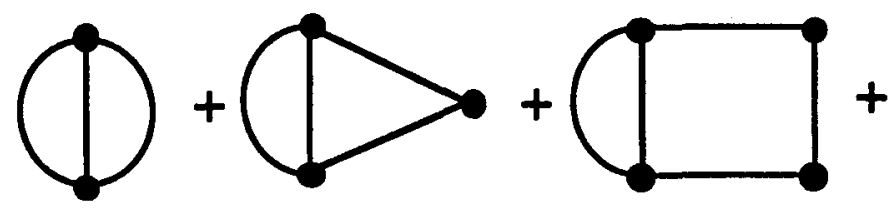

Fig. 2 Graphical illustration of the classical ladder sum for the OCP that screens the right hand rung of the three rung ladder diagram. Solid circles represent ions.

Continuing in this way provides an expansion for $S$, known as the Abe nodal (cluster) expansion (Abe 1959), that is valid at all values of the coupling. The result for the general case of a multi-component plasma is

$$
\begin{aligned}
& S=S_{\text {rine }}+\sum_{i j} S_{i j}+\sum_{i j k} S_{i j k}+\ldots \\
& S_{\text {ring }} \equiv S_{D H}=1 / 12 \pi \lambda_{D H}^{3} \\
& \lambda_{D}=\left(k T / 4 \pi e^{2} \sum_{i} Z^{2} \rho_{i}\right)^{1 / 2}
\end{aligned}
$$




$$
\begin{gathered}
S_{i j} \equiv S_{2}=\rho_{i} \rho_{j}\left[-B_{i j}\left(T, \lambda_{D}\right)-2 \pi \int_{0}^{-} d r r^{2}\left(q_{i j}-\frac{q_{i j}^{2}}{2}\right)\right] \\
S_{i j k} \equiv S_{3}=\rho_{i} \rho_{j} \rho_{k}\left[-B_{i j k}\left(T, \lambda_{D}\right)-\sum_{p=1}^{3} \iint d r_{i j} d r_{i k} q_{i k} q_{j k} f_{i j}\right. \\
\left.-\frac{2}{3} \iint d r_{i j} d r_{i k} q_{i j} q_{j k} q_{i k}\right]
\end{gathered}
$$

where

$$
q_{a b}=\frac{Z_{a} Z_{b} e^{-r / \lambda_{D}}}{k T r}
$$

is the Debye-Hückel screened potential, $\lambda_{\mathrm{D}}$ is the multi-component Debye length, and

$$
f_{a b}=e^{-q \omega}-1
$$

is the Mayer function.

Figure 3 shows the convergence of the Abe cluster expansion to Monte-Carlo simulations (Slattery, Doolen, and DeWitt 1982) of the one component plasma. The Debye-Hückel pressure term (corresponding to $\mathrm{S}_{\mathrm{DH}}$ ) is seen to have a very small range of validity. Significant discrepancies appear for $\Lambda>0.2$. The $S_{2}$ correction, analogous to the second virial coefficient for the Debye-Hückel potential, is somewhat of an improvement. The important point is that the Abe series offers a systematic way of calculating the EOS of the OCP. It was shown in Rogers (1981) that, if large numbers of Abe nodal terms are included, this convergence persists to large values of the coupling parameter, $\Gamma=Z^{2} e^{2} / a$. This shows that, contrary to some claims in the literature, that, when properly implemented, the Debye-Hückel potential is a valid plasma potential. Similar studies have been done for multicomponent mixtures of heavy ions (TCP) in a neutralizing background. Our interest here is limited mainly to weak to moderately coupled plasmas, but this example shows that the Abe procedure could be applied to plasmas occurring in white dwarf stars. Related examples of the convergence of the activity expansion method (see Section 7) to the OCP are given in Rogers and DeWitt (1973). They found that the convergence is somewhat faster in the 
activity expansion. Similar results have also been reported for electrolytic solutions (Wood, Lilley, and Thompson 1978).

Since electrons in real plasmas are always quantum mechanical, direct use of classical methods is not possible. Nevertheless, the main complications in real plasmas have to do with the highly classical long range correlations. The divergences associated with short range quantum interactions are easily handled by replacing the classical virial coefficients occurring in the $S_{j}$ with their quantum-mechanical equivalents (Rogers 1991; 1986; 1981; 1974; Rogers and DeWitt 1973). Quantum mechanical calculations for real reacting plasmas follow similar steps and the convergence should be similar to the example in Figure 3. In the current implementation the ion-ion correlations are included in all orders, but the electron-electron and electron-ion correlations are included only through $5 / 2$ order in the density (activity). Approximate methods for including electron-electron and electron-ion terms when even the electron-ion coupling is strong are given in Rogers 1979.

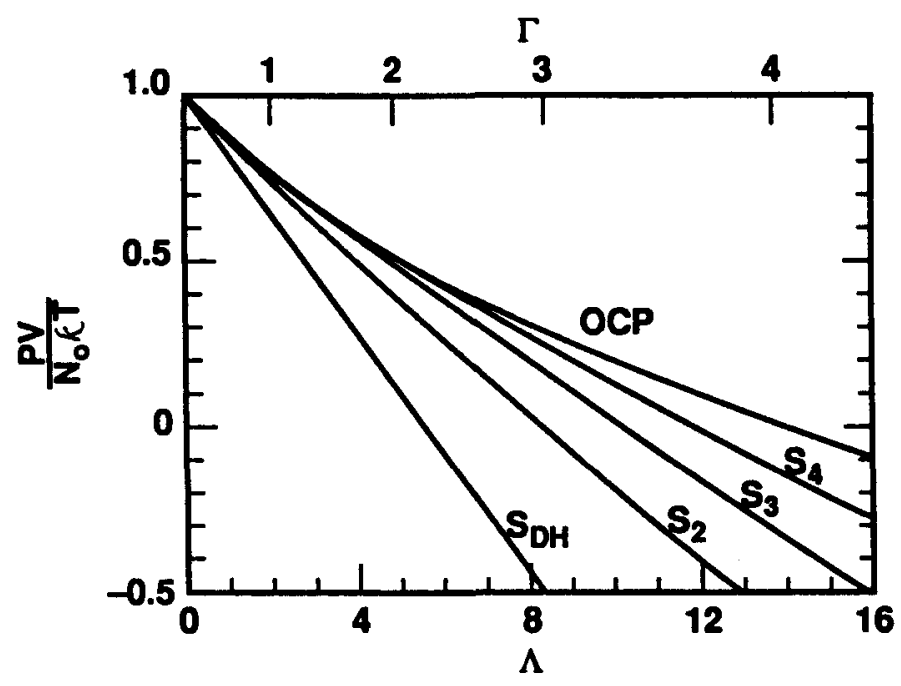

Fig. 3 Comparison of the Abe nodal expansion with Monte Carlo simulations of the OCP by Slattery, Doolen, and Dewitt (1982). Labels indicate the free energy terms used to calculate the pressure.

Elaborating on the remarks of the previous paragraph, we note that for a real plasma, involving electrons and ions, the diagrammatic sum that gives the Debye-Hückel correction equivalent to equation 16, involves additional terms corresponding to electron-electron and electron-ion interactions. 

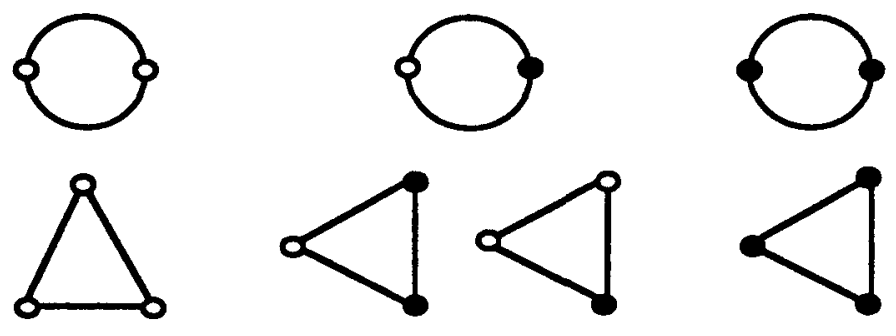

electron-electron

electron-proton

proton-proton

Fig. 4 Leading classical ring diagrams for an electron-proton plasma. Open circles represent electrons; closed circles represent protons.

The fact that electrons are now being treated as real particles, rather than a neutralizing fluid, introduces explicit diagrams in which electrons interact with electrons and diagrams in which electrons interact with ions. Due to the Coulomb repulsion the electron-electron terms are not divergent at small distances. However, quantum diffraction effects modify the result and can be treated by semi-classical methods. The classical electron-ion terms are divergent at small distances and can only be treated quantum mechanically; the large distance divergence is of course still classical. When $\gamma_{i j}$ is $<<1$, the ring sum free energy is

$$
S_{D H}^{c}=\frac{1}{12 \pi \lambda_{D}^{3}}
$$

where

$$
\lambda_{D H}=\left[\frac{k T}{4 \pi e^{2}\left(\rho_{c}+\rho_{p}\right)}\right]^{1 / 2}
$$

Equations $(28,29)$ are exactly the same as given by the classical theory (see equations (22-23)). This supports the earlier statement that the Coulomb correlations are largely classical. In general however, due to the quantum modifications at small distances, $\mathrm{S}_{\mathrm{DH}}$ is appreciably reduced when $\gamma_{i j}=\lambda_{i j} / \lambda_{D}>0.5$. For Boltzmann statistics the quantum corrected Debye-Hückel term can be written in the form

$$
S_{D H}=S_{D H}^{c} f\left(\gamma_{e e}, \gamma_{a i}\right)
$$


where $f(0,0)=1$. There are also some additional corrections due to exchange effects. The ion-ion diffraction parameter, $\gamma_{\mathrm{ii}}$, does not appear in equation (30), since $\gamma_{i i} \ll 1$ due to the mass of the ion.

Figure 5 displays the variation of $f$ with $\gamma_{e e}$ for the simple case of a non-degenerate electron gas for the "exact" calculations of Graboske and DeWitt (1974) and the approximate effective potential results of Rogers (1979).

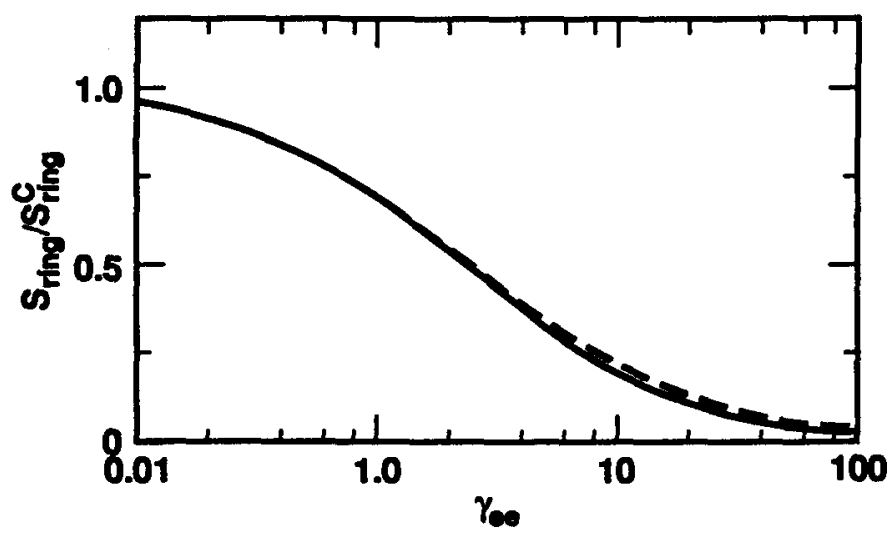

Fig. 5 Diffraction corrections to the electron-electron ring sum.

\subsection{EOS of a Hydrogen Plasma}

We have chosen the simple example of ionization equilibrium in a nondegenerate hydrogen plasma to compare the free energy obtained from a many body quantum statistical approach with a commonly used free energy minimization model, i.e.,

$$
e+p \rightleftarrows H
$$

The typical free energy minimization method would have

$$
F / k T=-N_{e} \ln \left(\frac{e g_{e}}{\rho_{e} \lambda_{e}^{3}}\right)-N_{p} \ln \left(\frac{e g_{p}}{\rho_{p} \lambda_{p}^{3}}\right)-N_{H} \ln \left(\frac{e g_{H}}{\rho_{H} \lambda_{H}^{3}} Z_{\text {int }}\right)-V S_{D H}^{c}
$$

where the first three terms on the right correspond to the translational free energies for electrons, protons, and hydrogen atoms, respectively, $Z_{\text {int }}$ is the sum over states (internal partition function), and $\mathrm{SBH}_{\mathrm{H}}$ is given by 
equation (28). As described in Section 2, some model must be introduced to make the sum in $Z_{\text {int }}$ finite. In practice this can be one of a number of possibilities such as the use of the energy levels of the Debye potential. Since $Z_{\text {int }}$ changes discontinuously at conditions where a state moves into the continuum and is no longer counted, equation (31) is not physically consistent.

The free energy that results from the many body diagrammatic approach is (Rogers 1991; 1989; 1986)

$$
F / k T=-N_{e} \ln \left(\frac{e g_{c}}{\rho_{e} \lambda_{e}^{3}}\right)-N_{p}\left(\frac{e g_{p}}{\rho_{p} \lambda_{p}^{3}}\right)-N_{H} \ln \left(\frac{e g_{H}}{\rho_{H} \lambda_{H}^{3}} Z_{\text {int }}^{p \ell}\right)-V S_{D H}
$$

where

$$
Z_{\text {in }}^{\mu d}=\sum_{n \ell}(2 \ell+1)\left(e^{-\beta E_{n \ell}}-1+\beta E_{n \ell}\right),
$$

which is closely related to $Z_{\mathrm{int}}^{\mathrm{e}}$ of equation (11), is the so called PlanckLarkin partition function. The sum in $\mathrm{Z}_{\mathrm{nt}}^{\mathrm{l}}$ ranges over the states in a screened potential which approaches the Debye-Hückel potential at very low density. As described in Rogers $(1986 ; 1981)$ the energy levels appearing in Z $\mathrm{lnt}$ are unscreened except for high lying states near the plasma continuum. The states that are screened change with plasma conditions. As a result $\mathrm{Z}_{\mathrm{nt}}^{\mathrm{pl}}$ is both finite and a continuous function of temperature and density; although the density dependence is very slight for normal stellar conditions. The MHD EOS displays a similar property through the use of the occupation probability formalism.

Figure 6 is an attempt to explain the result in equation (32). It shows (schematically) the contribution to the partition function for hydrogen as a function of principal quantum number at temperatures of 1-2 eV. Due to the large value of the Boltzmann factor at these temperatures, the ground state contribution is large. Because of the wide energy separation between $n=1$ and $n=2$, the contribution from $n=2$ has already dropped close to unity. For higher states the $n^{2}$ degeneracy causes the contribution to again increase. For the pure Coulomb potential the area under the curve becomes infinite and leads to the well known divergence of the atomic partition function. The high lying states are classical (closely spaced), which means that sums over quantum numbers can be replaced with integrals; while the low lying states are quantum mechanical. This suggests that the partition function can be separated into two parts; one requiring the explicit use of quantum numbers, the other only involving 
integrals as shown in the figure. The natural way to do this seems to be to use the analytic properties (see Section 3 ). The boundary between the two regions is of course fuzzy and not abrupt as actually shown. The discussion leading to equation (28) showed that, even though quantum mechanics is required to remove the divergence in the electron-ion terms, the ring sum is highly classical. This suggests that the electron-ion part of the ring summation leading to equation (28) has in some sense included the classical parts of $Z_{\text {int }}$. As a result, using the full $Z_{\text {int }}$ in equation (31) is incorrect, since it would double count much of the excited state part.

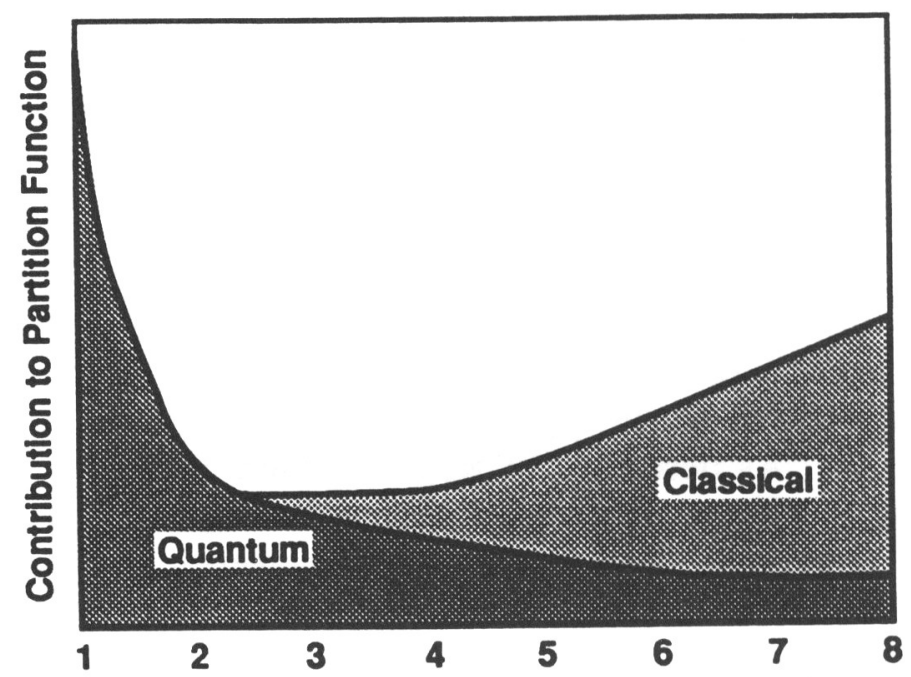

Fig. 6 Contributions to the hydrogenic bound state partition function. Lower shaded region corresponds to the quantum mechanical part; upper shaded region to the (approximate) classical part. The total is shown as a smoothed curve to emphasize the classical nature of the high n contribution.

The pressure for a partially ionized hydrogen plasma corresponding to equation (32), but also including degeneracy and exchange corrections, has the form

$$
P / k T=\frac{\rho_{\varepsilon} I_{3 / 2}\left(\alpha_{e}\right)}{I_{1 / 2}\left(\alpha_{e}\right)}+\rho_{p}+\frac{P_{c x}}{k T}+\frac{S_{D H}^{c}}{3} f_{p}\left(\gamma_{c e}, \gamma_{\varepsilon i}\right)
$$

where the $I_{n / 2}$ functions are the usual Fermi Functions, $\alpha_{e}=\mu_{e} / k T$ is the degeneracy parameter, 


$$
P_{e x}=\frac{2 \pi}{3 V} \int r^{2}\left(\frac{e^{2}}{r}\right)\left(g_{e \varepsilon}(r)-1\right) d r
$$

is the first order electron exchange, and $g_{e e}$ is the electron-electron distribution function for an ideal Fermi gas, the Debye length corrected for electron degeneracy is

$$
\lambda_{D}=\left[\frac{k T}{4 \pi e^{2}\left(\rho_{e} I_{-1 / 2}\left(\alpha_{e}\right) / I_{1 / 2}\left(\alpha_{e}\right)+\rho_{p}\right)}\right]^{1 / 2}
$$

and $f_{p}\left(\gamma_{\varepsilon \varepsilon}, \gamma_{e i}\right)$ is the diffraction correction to the pressure similar to Figure 5. There are some additional exchange corrections to equation (34), which are not explicitly shown. The first order exchange correction (equation (35)) is frequently omitted in astrophysical EOS calculations, but in view of the current need for high precision that is no longer acceptable.

The origin of the exchange term is easily seen from the electronelectron distribution function for an ideal gas, as shown in Figure 7.

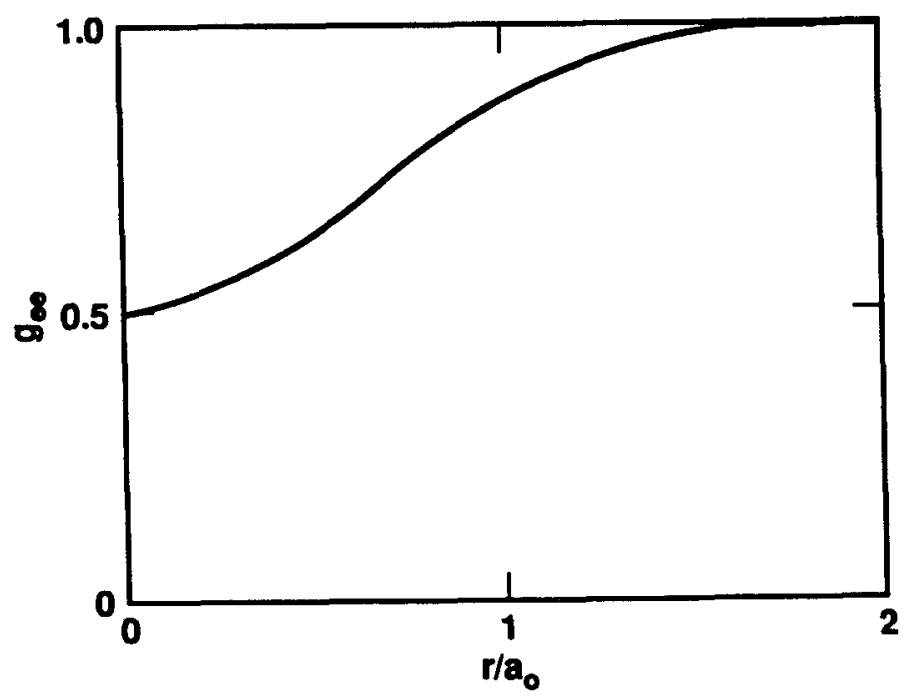

Fig. 7 Schematic representation of the ideal Fermi gas electron-electron distribution function.

For an ideal Boltzmann gas, gee is everywhere unity, as is $\mathrm{g}_{\mathrm{pp}}$ ang $\mathrm{gep}_{\text {epot }}$ shown), so that, the first order contribution to the pressure, i.e., the average over $\mathrm{Z}_{\mathrm{i}} \mathrm{Z}_{\mathrm{j}} \mathrm{e}^{2} / \mathrm{r}$, is exactly zero. However, when the electron 
distribution is allowed to redistribute itself, due to the Pauli repulsion, gee is forced to have the value $1 / 2$ at $r=0$ and gradually increases to unity as $r$ $>$ a0. This produces a small, negative, non-cancellation in the average of $\mathrm{Z}_{\mathrm{i}} \mathrm{Z}_{\mathrm{j}} \mathrm{e}^{2} / \mathrm{r}$. In other words the quantum statistical effects that produce the degeneracy corrections are always partially reduced by the Coulomb interactions. The presence of the Coulomb interaction also affects the electronic charge distribution, which produces additional corrections to the first order average over $\mathrm{Z}_{\mathrm{i}} \mathrm{Z}_{\mathrm{j}} \mathrm{e}^{2} / \mathrm{r}$. This leads to what are known as second order (or higher) exchange corrections.

The Fermi function ratios appearing in equation (34) are shown schematically in Figure (8) as a function of the degeneracy parameter. The ratio of $I_{3} / 2 / I_{1} / 2$ increases with increasing degeneracy, indicating that degeneracy increases the electron contribution to the pressure, while the ratio $\mathrm{I}_{1 / 2} / \mathrm{I}_{1 / 2}$ decreases, indicating that highly degenerate electrons do not contribute to the screening.

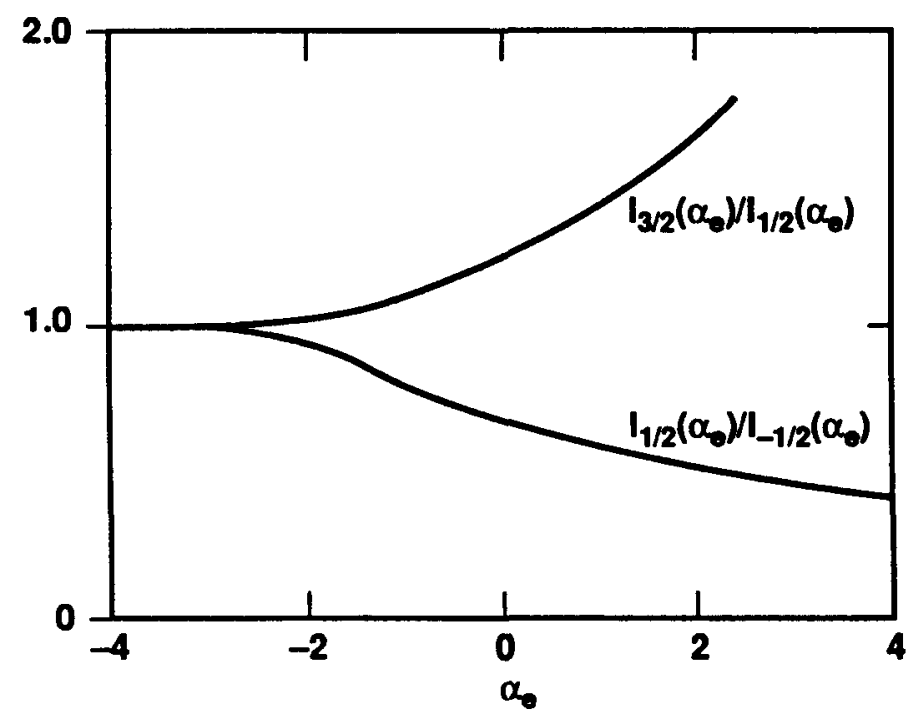

Fig. 8 Fermi function ratios appearing in equation (34).

\subsection{The Activity Expansion Method}

A many body Quantum statistical procedure for calculating the EOS of reacting multi-component plasmas is described in Rogers (1991). A somewhat different procedure, specialized to hydrogen plasmas is given in Bartsch and Ebeling (1971). These approaches treat the plasma in terms of its electrons and nuclei and are thus "physical picture" methods. 
Composite particles, i.e., ions, atoms, and molecules, arise naturally in the physical picture, such that, plasma screening effects on the bound states are determined from theory. This is a definite advantage over chemical picture methods in current use; all of which introduce models to obtain these effects.

For a discussion of the activity expansion method, as developed for multi-component plasmas, the reader is referred to Rogers (1991). The main steps in the method can be summarized as follows

I. Start from the classical multicomponent canonical ensemble.

II. Carry out the Abe reorganization in powers of $\beta u$ (see Section 4) to obtain a finite expression for $S\left(T,\left\{\rho_{i}\right\}, \lambda_{D}\right)$. The reason for this step is that the analogous summation in the grand-canonical ensemble gives $S\left(T,\left\{z_{i}\right\}, \lambda\right)$, i.e., exactly the same analytic function except density is replaced by the activity; where

$$
z_{i}=\left(2 s_{i}+1\right) \lambda_{i}^{3} \exp ^{\mu_{i} / k T}
$$

is the activity, $\mathrm{i}=\left\{e, \alpha_{\mathrm{j}}\right\}, \alpha_{\mathrm{j}}$ is an atomic nucleus of charge $\mathrm{Z}_{\mathrm{j}}$, and $\lambda$ is a screening length that approaches $\lambda_{D}$ when the Coulomb coupling is weak.

III. Construct a generating function method for developing the corresponding grand canonical ensemble from functional derivatives of $S\left(T,\left\{z_{i}\right\}, \lambda\right)$. The advantage of this procedure is that, as a result of the resummation in step $I, S\left(T,\left\{z_{i}\right\}, \lambda\right)$ is divergence free and therefore so is the grand canonical expression.

IV. Recollect terms to introduce screened cluster coefficients; i.e. construct the grand canonical ensemble analogs to equations (2127). At this point all states are screened by the plasma.

V. Replace all the classical Boltzmann factors in the classical grand canonical ensemble with $\operatorname{Tr} \exp ^{-\beta H}$.

VI. Introduce an augmented set of activity variables to account for the formation of ions, atoms, and molecules; i.e., construct composite particle activities from products of $z_{e}$ and $z_{\alpha}$. This renormalization removes the screening of low lying, bound states that were initially present after step IV. 
VII. Reorganize the resulting activity expressions to take advantage of the charge asymmetry of high $Z$ ions. Since the ion-ion terms are classical, it is possible to include them in all orders. Limitations on the range of validity are set by the electron-ion diagrams, which require quantum mechanics and are only included to $5 / 2$ order in the coupling parameter.

This procedure will only recover the linear correction in $\gamma_{i j}$ in the expansion of $\mathrm{f}\left(\gamma_{\mathrm{ee}}, \gamma_{\mathrm{ei}}\right)$. However, it is possible to introduce additional corrections using known results from other sources (Rogers 1981). Since the activity expansion approach is based on a systematic method, it is possible to determine its range of validity. This is shown in Figure 9 for an argon plasma which shows contours of constant $\Lambda=\left\langle Z^{2}>e^{2} / k T \lambda_{D}\right.$. As already shown in Figure 3 for the classical OCP, the contour $\Lambda=0.2$ is the approximate limit of validity of the Debye-Hückel theory. The current version of the code includes all orders of ion-ion interactions, but only terms through $5 / 2$ order in the activity for electron-electron and electronion interactions. Consequently, in the shaded region on the right side of the figure the errors in the pressure are estimated to exceed $5 \%$. The limitation at the low temperature end of this region is due to the inclusion of only two particle neutral-neutral and ion-neutral interactions.

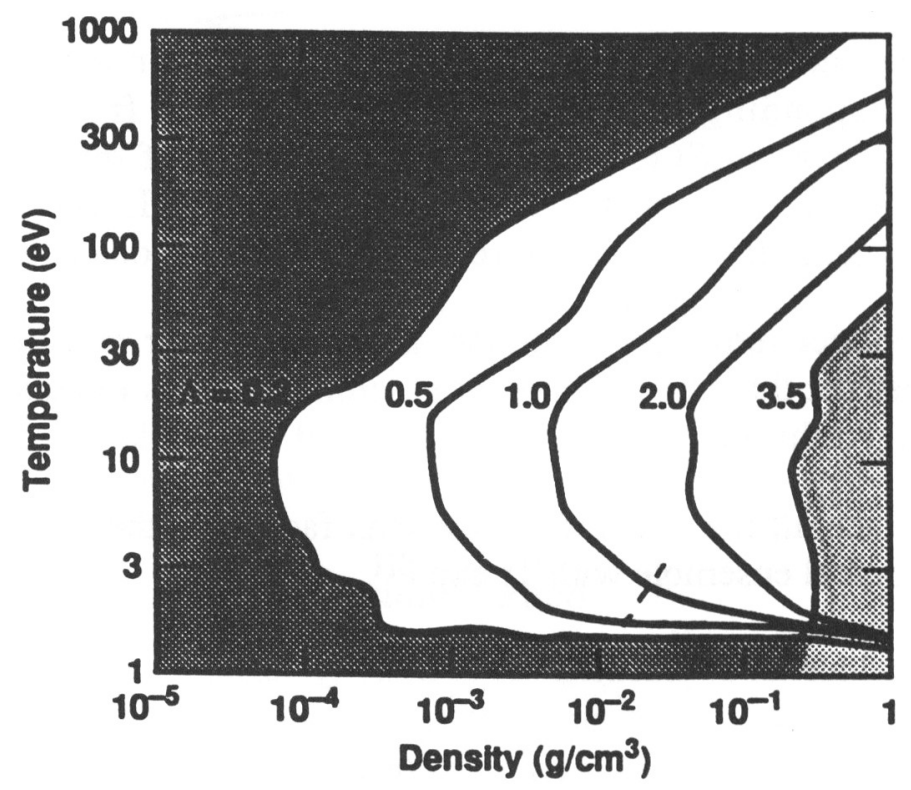

Fig. 9 Contours of constant $\Lambda=\left\langle Z^{2}>e^{2} / k T \lambda_{D}\right.$ for an argon plasma. 
The short dashed line in the lower right hand part of Figure 9 is the approximate location of some recent shock wave EOS measurements by Erskine, Rosznyai, and Ross (1994). Figure 10 compares this experimental work with the Saha equation and the activity expansion method. The coupling parameter, $\Gamma$, is around unity and the temperature is $2.5 \mathrm{eV}$ at the densest point. The activity expansion method is in good agreement with the experiment. Additional experimental work is in progress.

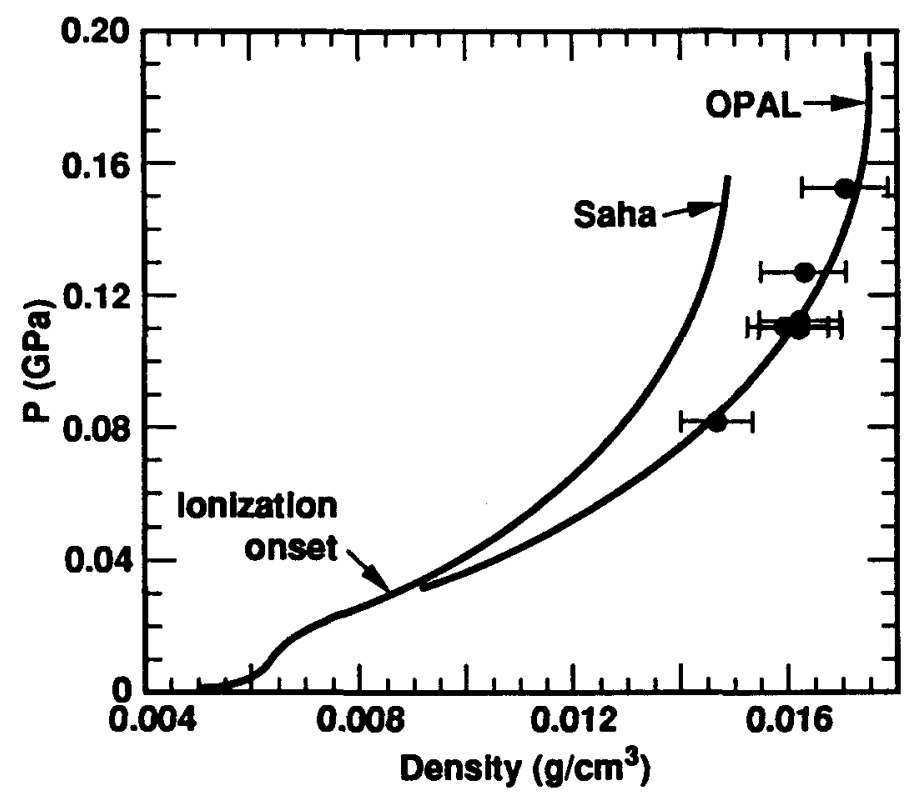

Fig. 10 Comparison of the activity expansion method (curve labeled OPAL) with the shock wave experiments of Erskin, Rosznyai, and Ross (1994). To demonstrate the importance of Coulomb interations, the simple Saha equation is also shown.

\subsection{Equation of State Comparisons}

The EOS properties frequently needed in stellar modeling are the first order thermodynamic quantities and the following second order quantities (Cox and Guili 1968)

$$
\chi_{T}=\left(\frac{\partial \ln P}{\partial \ln T}\right)_{\rho}, \quad \chi_{\rho}=\left(\frac{\partial \ln P}{\partial \ln \rho}\right)_{T}
$$




$$
\begin{aligned}
& \Gamma_{1}=\left(\frac{\partial \ln P}{\partial \ln \rho}\right)_{S}, \quad \frac{\Gamma_{2}}{\Gamma_{2}-1}=\left(\frac{\partial \ln P}{\partial \ln T}\right)_{S} \\
& C_{V}=\left(\frac{\partial E}{\partial T}\right)_{V}
\end{aligned}
$$

Comparisons of the several EOS methods described above have been carried out by Däppen (1992). A few selected examples are repeated here. Figure 11 compares results for $\chi_{\mathrm{T}}$. Figure 11 a shows that for stellar envelope conditions, that there is only a few percent difference between the EFF and MHD approaches. Figure $11 \mathrm{~b}$ shows that the differences between the MHD and OPAL equations of state are much smaller than their differences with EFF. Similar results were found for the other second order quantities. The seismic modes are determined only by the deviations of $\Gamma_{1}$ from the ideal gas value $5 / 3$. Consequently, differences similar to those shown in Figure $11 \mathrm{a}$ for $\chi_{\mathrm{T}}$ substantially affect the agreement with the p-mode data. The MHD and OPAL equations of state are in somewhat better agreement with observational data than is the simple EFF model (Christensen-Dalsgaard and Däppen 1993).

At higher densities, where the Coulomb coupling is outside the range of validity of the Debye-Hückel theory, differences between MHD and OPAL become significant. Figure 12 shows a comparison of $\Gamma_{1}$ along an isochore having density $0.1 \mathrm{gm} / \mathrm{cm}^{3}$. The region around two million degrees corresponds to conditions near the bottom of the solar convection zone. Differences in the two EOS methods are fairly small in this important solar region. However, substantial differences occur at temperatures of a few hundred thousand degrees. These differences could effect the modeling of very low mass stars.

In a recent paper Dziembowski, Pamyatnykh, and Sienkiewicz (1992) used helioseismological data to test the MHD equation of state. They found evidence that this approach is inadequate for conditions that exist in the fractional solar radius range $r / R=0.85$ to 0.95 . We have used the activity expansion method (Section 7) to study two points in this region (Rogers and Iglesias 1993). One point is located at $\mathrm{r} / R=0.88, \mathrm{~T}=744,000$ $\mathrm{K}$ and $\rho=0.0348 \mathrm{~g} / \mathrm{cm}^{3}$; the other is at $\mathrm{r} / R=0.90, \mathrm{~T}=612,000 \mathrm{~K}$ and $\rho=0.0259 \mathrm{~g} / \mathrm{cm} 3$. We were able to draw several conclusions (see Figure 1 of Rogers and Iglesias 1993). 1) The activity expansion result is appreciably different than the MHD result for $\Gamma_{1}$, on the fine sensitivity 

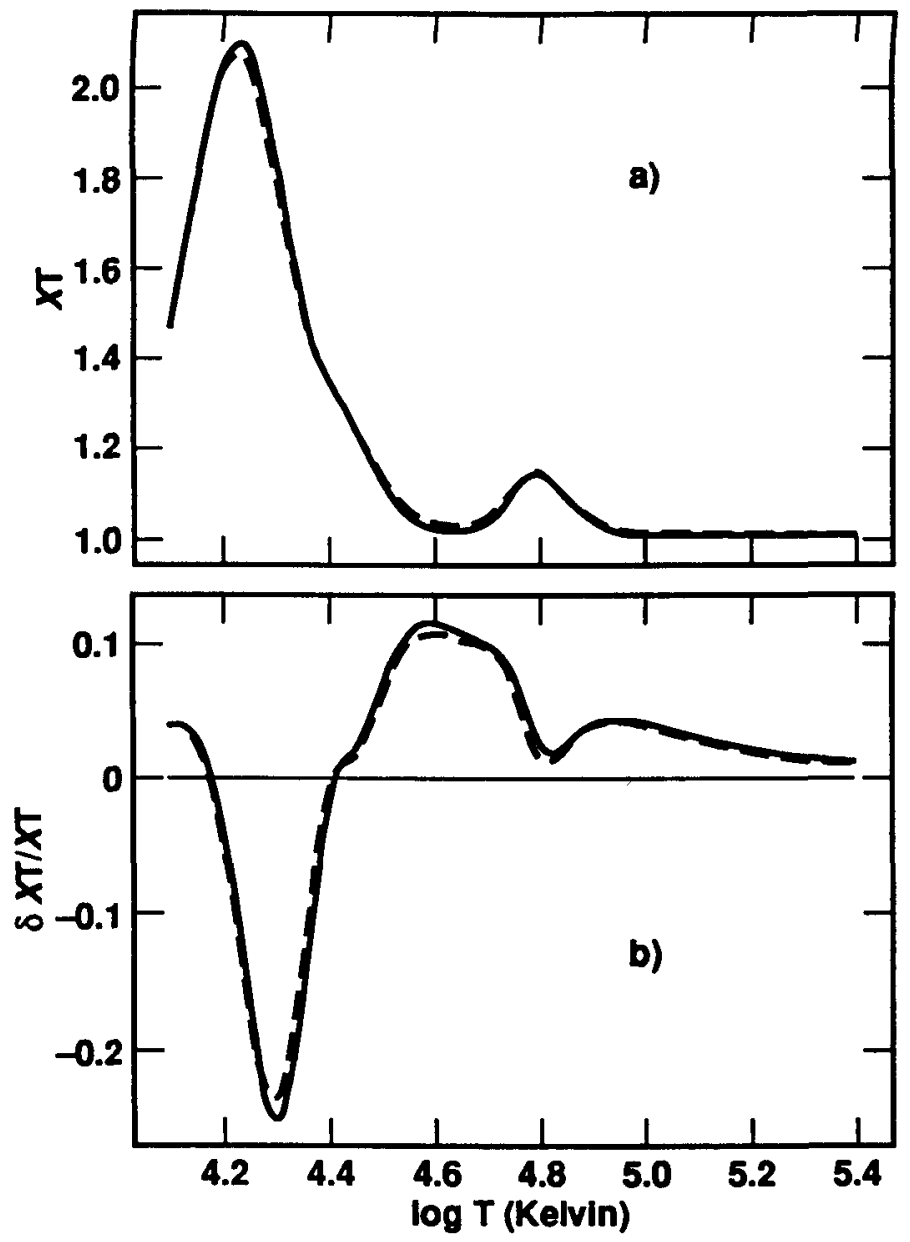

Fig. 11 Comparison of $x_{T}$ on an isochore with $p=10^{-5.5}$. Part a compares the EFF and MHD equations of state; solid line EFF and the dashed line MHD. The mixture is $90 \% \mathrm{H}$ and $10 \% \mathrm{He}$ by number abundance. OPAL is indistinquishable from MHD on this scale. Part $b$ compares the MHD and OPAL equations of state by looking at the relative differences with EFF: i. e., $\left(x_{\mathrm{T}}\right.$ opal $\left.x_{\mathrm{T}} \mathrm{EFF}\right) x_{\mathrm{T}} \mathrm{EFF}$ (solid line) and $\left(x_{\mathrm{T}}^{\mathrm{MHD}} \chi_{\mathrm{T}}{ }^{\mathrm{EFF}}\right) x_{\mathrm{T}} \mathrm{EFF}$ (dashed line). [From Dappen (1992) with permission]. 


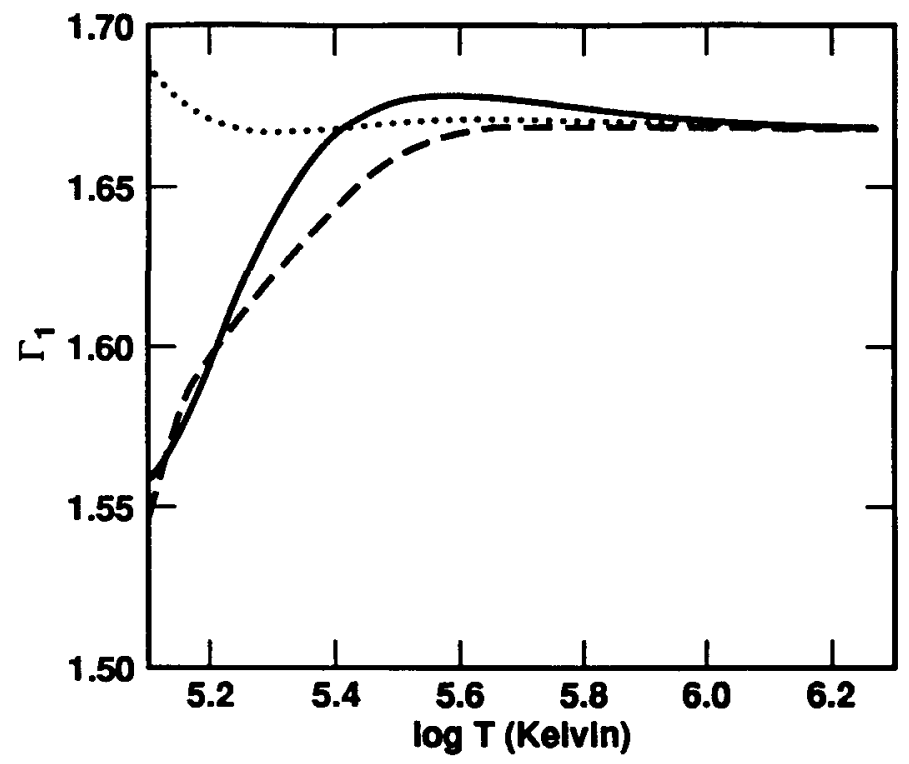

Fig. $12 \Gamma_{1}$ for $\rho=0.1 \mathrm{~g} / \mathrm{cm}^{3}$ for the mixture of Fig. 11; solid line CEFF; dashed line MHD: dotted line OPAL equation of state. [From Dappen (1992) with permission ]

scale of the experimental data; 2) the Coulomb corrections are important; 3 ) the metallicity has a surprisingly large effect on $\Gamma_{1}$; and 4) the composition of $Z$ is important. For example, we found that if we assume $\mathrm{Z}$ is composed entirely of $\mathrm{Si}$, that a close match of the inversion data is obtained with just solar metallicity $(0.0193)$.

\subsection{OPAL Equation of State Tables}

The inability to resolve a number of long standing discrepancies between theory and observation through improved modeling led to the speculation that the widely used Los Alamos opacities were missing important sources of opacity in the $10^{5}-10^{6} \mathrm{~K}$ temperature range (Simon 1982). Due to this speculation and the need for the opacity of low $Z$ materials to model laser produced plasmas, the OPAL opacity effort was undertaken (Iglesias, Rogers, and Wilson 1987; Rogers and Iglesias 1992; Iglesias and Rogers 1993). While it was necessary to calculate the occupation numbers as part of this effort, the much greater accuracy and table density required to calculate derivatives of the equation of state, 
made it necessary to defer these calculations. The success of the OPAL opacities in helping to improve theoretical models (Guenther 1992) has made it essential to also provide EOS data that is consistent with the opacity tables. We now have available EOS tables for the following conditions:

Grevesse 1991 mixture (Rogers and Iglesias 1992)

$0.005>\mathrm{T}_{6}<100$

$$
\begin{aligned}
& 10^{-14}>\rho<10^{7} \mathrm{~g} / \mathrm{cm}^{3} \\
& X=0,0.2,0.4,0.6 \text {, and } 0.8 \\
& Z=0,0.02 \text {, and } 0.04 \\
& Y=1-X-Z
\end{aligned}
$$

Data has been tabulated for the quantities discussed in Section 8.

Figure 13 shows $\Gamma_{1}-5 / 3$ vs. $\quad T_{6}$ at several values of $\log R$ for a simple mixture of $\mathrm{H}$ and $\mathrm{He}$. Figure 13a shows results for the full range of the tables; while Figure $13 \mathrm{~b}$ is limited to the high temperature range above $100,000 \mathrm{~K}$. The large negative deviations from $5 / 3$ at low temperature in Figure 13a is due to placing energy into the formation of ions and atoms. In the few hundred thousand degree range the negative deviation due to bound complexes is competing with the positive deviations caused by Coulomb interactions. The positive Coulomb deviations start to dominate with increasing values of $\log R$.

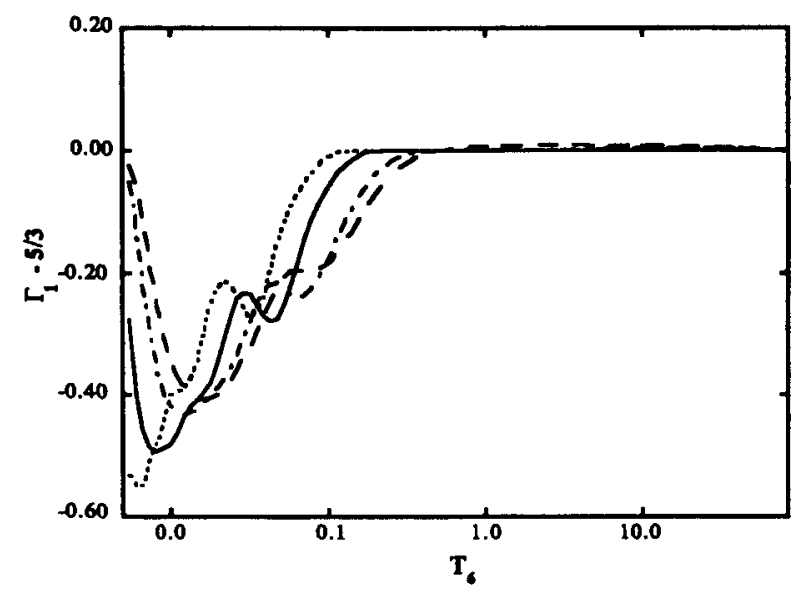

Fig. 13 (a) $\Gamma_{1}-5 / 3$ vs. $T_{6}$ for hydrogen mass content $X=0.4$ and helium mass content $Y=0.6$. Dotted line $\log R=-5$ : solid line $\log R=-3$; dot-dash $\log R=-1$; dashed line $\log R=0$. 


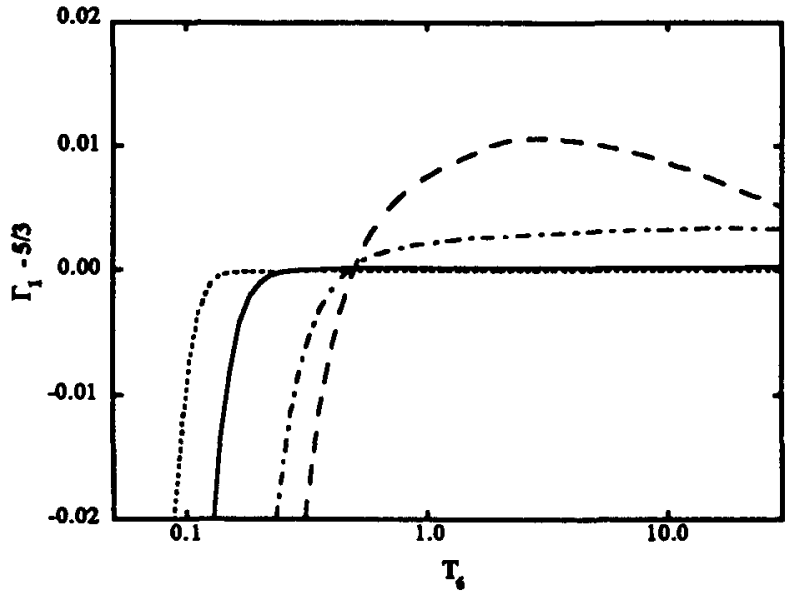

Fig. 13 (b) Same as (a) except for reduced temperature range.

Figure 13c is similar to Figure 13b, but for the Grevesse 1991 mixture. The small amount of high $\mathrm{Z}$ admixture has a noticeable effect on the results. It is apparent that the modeling results will be sensitive to uncertanties in the abundances of heavy elements, e.g. neon in the million degree range.

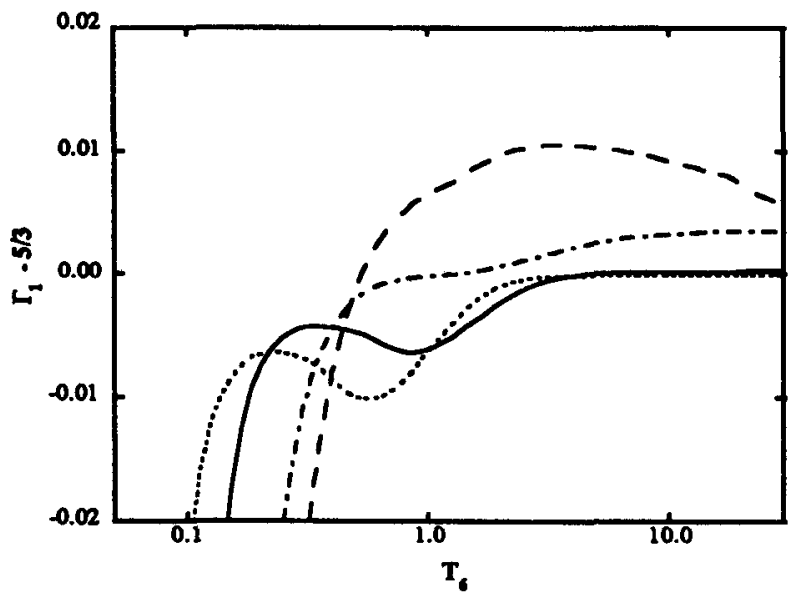

Fig. 13 (c) Same as (b) with $X=0.4, Y=0.58$ and $Z=0.02$. 
Work performed under the auspices of the U.S. Department of Energy by the Lawrence Livermore National Laboratory under contract number W-7405-ENG-48.

\section{References}

Abe, R., 1959, Theor. Phys. 22, 213

Alustuey, A., 1994, this volume

Alustuey, A. and Perez, A., 1992, Europhys. Lett. 1, 13

Bartsch, G. P., and Ebeling, W., 1971, Beitr. Plasma Phys. 11, 393

Beth, E. and Uhlenbeck, G. E., 1939, Physica 4, 915

Bolle, D., 1987, Phys. Rev. A36, 3259 , 1989, Phys. Rev. A39, 2752

Cox, J. and Guili, R., 1968, Stellar Structure (Gordon and Breach, New York)

Christensen-Dalsgaard, J. and Däppen, W., 1993, Astron. Astrophys. Rev. 4, 267

Christensen-Dalsgaard, J. and Däppen, W., 1991, in Challenges to Theories of the Structure of Moderate-Mass Stars, eds. D. O. Gough and J. Toomre (Lecture Notes in Physics, 388, Springer, Heidelberg)

Däppen, W., Anderson, L. S., and Mihalas, D., 1987, Ap. J. 319, 195

Däppen, W., 1992, Rev. Mexicana Astron. \& Astrof. 23, 1144

Dashen, R., Ma, S. K., and Bernstein, H. J., 1969, Phys. Rev. 187, 345

DeWitt, H., E., 1966, J. Math. Phys. 7, 6169

Dziembowski, W. A. , Pamyatnykh A. A., and Sienkiewicz, R. , 1992, Acta Astronomica 14, 5

Ebeling, W. , 1974, Physica 73, 573

Ebeling, W., Kraeft, W. D., and Kremp, D., 1977, Theory of Bound States and Ionization Equilibrium in Plasmas and Solids (Berlin, AkademieVerlag; New York, Plenum)

Eggelton, P. , Faulkner, J. and Flannery, G. P., 1973, A\&A 23,,261

Erskine, D., Rosznyai, B., and Ross, M., 1994, JQSRT 50

Goldsmith, S., Griem, H. E., and Cohen, L., 1984, Phys. Rev. A30, 2775

Graboske, H. C., and DeWitt, H. E., 1974 (unpublished)

Guenther, D. B, 1992, Nature 359, 585

Hill, T. L., Statistical Mechanics (McGraw-Hill, New York, 1956), Chap. 5

Hummer, D. G., and Mihalas, D., 1988, Ap. J. 331, 794

Iglesias, C. A., Rogers, F. J., and Wilson, B. G., 1987, Ap. J. (Letters), 322, L45

Iglesias, C. A. and Rogers, F. J., 1993, ApJ 412, 752

Kraeft, W. D., Kremp, D., Ebeling, W., and Röpke, G., 1986, Quantum Statistics of Charged Particle Systems, (Plenum Press, New York)

Krasnikov, Yu. G., and Kucherenko, V. I., 1978, Teplofiz. Vys. Temp. 16, 43

Krasnikov, Yu. G., 1977, Sov. Phys. JETP 46, 271 
Larkin, A. I., 1960, Sov. Phys. JETP 11, 1363

Levinson, N., 1949, Danshe Mat. Fys. Medd, No. 25

Mayer, J. E., 1950, J. Chem. Phys. 18, 1426

McChesney, M., 1964, Can. J. Phys. 42, 2473

Pisano, C., and McKellar, B. H. J., 1989, Phys. Rev. A40, 6597

Rogers, F. J., 1974, Phys. Rev. A10, 2441 , 1977, Phys. Lett. 61A, 358

, 1979, Phys. Rev. A19, 375

, 1981, Phys. Rev. A24, 1531

1984, Phys. Rev. A29, 868

, 1986, Ap. J. 310, 723

1990, Ap. J 352, 689

Rogers, F. J., 1991, in High Pressure Equations Of State: Theory and

Applications, ed. S. Eliezer \& R. A. Ricci (North Holland, New York, 1991)

Rogers, F. J. and DeWitt, H. E., 1973, Phys. Rev. 8, 1061

Rogers, F. J., Graboske, H. C., and DeWitt, H. E., 1971, Phys. Lett. 34A, 127

Rogers, F. J. and Iglesias, C. A., 1991, ApJS 79, 507

Rogers, F. J. and Iglesias, C. A., 1993, A.S.P. Conf. Ser., V42, Ed. Timothy M. Brown

Saha, M., 1920, Phil. Mag. 40, 472

Saumon, D., and Chabrier, G., 1991, Phys. Rev. A44, 5122 1992, Phys. Rev. A46, 2084

Sevastyanenko, V., 1985, Beitr Plasmaphys. 25, 151

Simon, N. R., 1982, ApJ 269, L87

Slattery, W, L., Doolen, G. D., and DeWitt, H. E., 1982, Phys. Rev. A26, 225

Swenson, F. J., and Rogers, F. J., 1994, (in preparation)

Swenson, F. J., VandenBerg, D. A., Alexander, D. R., and Irwin, A., 1994, (in preparation)

Uhlenbeck, G. E., and Beth, E., 1936, Physica 3, 729

Wood, R. H., Lilley, T. H., and Thompson, 1978, J. Chem. Soc. Far. Trans. I 74, 1301 